\title{
Hope, Optimism and Its Relation to Suicidal Ideation among University Students
}

\author{
Rajkumar E. ${ }^{1 *}$, Vishwanatha B N. ${ }^{2}$, Hemilnath. E G. ${ }^{2}$
}

\section{ABSTRACT}

Suicide is very important issue around the world. In India, adolescent suicide constituted $17 \%$ of the overall reported suicide cases in the world. Suicidal behavior and ideation among adolescents exhorts considerable attention. Variety of factors has been linked to suicidal ideation, only few studies have examined role of hope and optimism among adolescents population. This study intended to examine the relationship between hope and optimism and suicidal ideation among university students. The study adopted correlation design with the independent variable being hope, optimism and dependent variables suicidal ideation. The sample was drawn from the adolescents aged between 18 to 23 years from central university of Karnataka. Gulbarga. The sample consisted of 70 adolescent students. Convenient sampling technique method was used to select the participants. The results indicated there is no statistically significant relationship between hope and suicidal ideation. There is a statistically significant negative between Optimism and suicidal ideation.

Keywords: Hope, Optimism, Suicidal Ideation, Students

Suicide is an epidemic health concern. Worldwide, nearly one million deaths are caused by suicide (WHO, 2012). About 800,000 people commit suicide worldwide every year (World Health Organization 2012) of these 135,000 (17\%) are residents of India (The Registrar General of India, Government of India 2012) a nation with 17.5\% of world population. Between 1987 to 2007, the suicide rate increased from 7.9 to 10.3 per 100,000 (Vijaykumar 2007), with higher suicide rates in southern and eastern states of India (Polgreen, 2010). In 2012, Tamil Nadu (12.5\% of all suicides), Maharashtra (11.9\%) and West Bengal (11.0\%) had the highest proportion of suicides (The Registrar General of India, 2012) among large population states, Tamil Nadu and Kerala had the highest suicide rates per 100,000 people in 2012. The male to female suicide ratio has been about 2:1 (The Registrar General of India, (2012) In India, about 46,000 suicides occurred each in 15-29 and 30-44 age groups in 2012 - or about 34\% each of all suicides ( The Registrar General of India, 2012). So studying suicidal ideation becomes imploratory. Positive psychological approach identifies factors which buffers and act as preventive mechanism against suicidal behaviour complementing traditional approach of

\footnotetext{
${ }^{1}$ DST -JRF, Department of Psychology, Central University Of Karnataka, Karnataka

${ }^{2}$ M.Sc Psychology, Central University Of Karnataka, Karnataka

*Corresponding Author

(C) 2015 I Rajkumar, Viswanath B, Hemilnath E; licensee IJIP. This is an Open Access Research distributed under the terms of the Creative Commons Attribution License (http://creativecommons.org/licenses/by/2.0), which permits unrestricted use, distribution, and reproduction in any Medium, provided the original work is properly cited.
} 
tracking risk factors (Wingate et al, 2006). Suicidal ideation and suicidal behaviour among students in the age of adolescence is a worldwide problem that exhorts considerable attention. Life for many adolescents is not easy and adolescence is considered by many as period of storm and stress.

Developmental factors intertwine with the conflicting demands like High level of parental expectations, academic demands, adjustment to new situations, school and college environment, peer pressure; managing relations are most common events in the life of a adolescent which are consequential to suicidal behaviour.

Contemporary psychologists are more concerned about adolescent problems aroused by high level of parental expectations, academic demands, adjustment problems in new situations, school and college environment, and peer pressure, physical and mental changes and growth.

Researchers have tried to understand the suicide phenomenon by examining the potential contributions of psychological factors to suicidal thoughts and behaviors. Mainly research has focused upon psychopathological risk factors of suicidal thoughts and behavior but less attention paid on protective factors which focus on pathology rather than on strength and resiliency (Seligman \& Csikszentmihalyi, 2000). Obviously, it is important to do research on risk factors for suicidal behavior, at the same time considering factors, which may protect against suicidal behavior, with the ultimate goal of preventing needless injuries and deaths.

Scheier and Carver (1985) defined optimism as expecting good things to happen in One's future. Optimists have a more general expectation of good rather than differentiating things happening whether it is through their own actions, the actions of others, or through outside forces. Optimism seems particularly worthy of investigation as a possible protective factor against suicidal thoughts and behaviour. Optimism, as defined by Scheier and Carver (1985), has its roots in the control theory model of self-regulation. Inspite of the many studies investigating optimism and its relation to various forms of Distress. Hirsch, Conner, and Duberstein (2007) found that optimism negatively predicted suicidal ideation in a college student sample, after accounting for the effects of both depression and hopelessness. Hirsch and colleagues then concluded that cultivating positive outcome expectancies may be useful as a suicide prevention approach. Hirsch and Conner (2006) noted no significant interaction effect between dispositional optimism and hopelessness in predicting suicidal ideation. In contrast, a study by Hirsch, Wolford, LaLonde, Brunk, and Morris (2007) found a significant moderating effect between optimism and negative life events, such that higher levels of optimism provided individuals with less protection against suicidal ideation and attempts as the number of negative life events increased.

Hope is a perception of reachable goals in both pathway and agent thoughts (Snyder et al., 2000). Achievability of specific goals has to have a plan with sensible amount of goal-directed energy. 
Snyder explained that goal is a cognitive aspect of hope in which enables the mental action sequences. High hope individuals can generate alternate routes to achieve particular goals as compared to low hope individuals. Agency thoughts motivate and cater mental energy to initiate and pursue using a pathway to reach specific goals (Snyder, 2002). Research shows that hope as being the protective factor of suicidality. Davidson and Wingate (2013) found that hope can predict lower levels of suicidal risks in a clinical sample. They found that hope only reduced one's level of burdensomeness and dimnished belongingness but not suicidal ideation. Beck, Kovacs, and Weissman (1979) describes suicidal ideation is consequential to suicidal behaviour and defined it as the potential indicator for the same. So studying suicidal ideation becomes imploratory.

The purpose of the present study was to examine the relationship between hope, optimism and suicidal ideation among university students. And it was hypothesized that there will be a significant negative relationship between hope, optimism and suicidal ideation.

\section{METHOD}

The design of the study is correlation design with the independent variable being hope, optimism and dependent variables suicidal ideation. The sample was drawn from the adolescents aged between 18 to 23 years from central university of Karnataka, Gulbarga. The sample consisted of 70 adolescent students. Convenient sampling technique method was used to select the participants. The inclusion criteria for the sample were that Students, who are UG and PG with no Psychiatric problems, and will be able to give informed consent. The exclusion criteria for the sample were the students who are having psychiatric problems and who are not giving informed consent.

\section{Tools:}

1) Optimism- Life Orientation Test- revised.( Scheier, Carver, \& Bridges (1994).

This test has 10 items to asses' optimism. Each item in the test has five responses in the form of 5-point Likertscale ranging from Strongly Disagree, Disagree, Uncertain, strongly agree. The six non filler items in the test are added to produce an overall score. The questionnaire life Orientation Test is From Scheier, Carver, \& Bridges (1994). It is Copyright (1994) by the American Psychological Association Reproduced with permission.

\section{Adult Dispositional Hope Scale (Snyder, Irving, \& Anderson, 1991).}

The adult hope scale (AHS) measures Snyder's cognitive model of hope which defines hope as "a positive motivational state that is based on an interactively derived sense of successful (a) agency (goal-directed energy), and (b) pathways (planning to meet goals)" (Snyder, Irving, \& Anderson, 1991). The adult hope scale contains 12 items to asses hope. Each item in the scale is having four responses ranging from definitely false, mostly false, mostly true, and definitely true. Four items measure pathways thinking, four items measure agency thinking, and four items are fillers. 


\section{Suicidal Ideation Scale (SIS-SDBV):}

The suicidal ideation scale developed by Devendra Singh sisodia and Vibhuti Bhatnagar used for assessing suicidal ideation. The scale was developed by using likert technique with an aim to measure suicidal ideation. The resent scale has 25 statements out of which 21 are positive statements and 4 are negative statement. The reliability of the scale was determined by (a) $\mathrm{T}$ test method and (b) internal consistency method. The test-retest reliability was 0.78 and consistency value for the scale is 0.81 .The scale is high in content validity. The scale was validated against the external criteria and coefficient obtained was 0.74 .

\section{Procedure of Data Collection}

Before proceeding to the data collection, the consent of subjects was taken, rapport was established to make them comfortable. The researcher introduced himself and explained the purpose of his research to students. Then researcher circulated the copies of questionnaires to students. Subjects were instructed to go through the instructions written in the questionnaires before answering the questions. After data collection, scoring of the responses was done according to the scoring procedure given for each tool.

\section{RESULTS:}

Table 1 Showing the Spearman correlation of Hope Optimism and Suicidal Ideation

\begin{tabular}{|lccc|}
\hline & Suicidal ideation & M & SD \\
\hline Optimism & $-288^{*}$ & 33.80 & 4.94 \\
Hope & -.157 & 35.64 & 3.72 \\
\hline
\end{tabular}

${ }^{*} p<.05, * * p<.01$

The above table shows co relation between hope, optimism and suicidal ideation. The results indicated there is no significant relationship between hope and suicidal ideation $(\mathrm{M}=35.6$; $\mathrm{SD}=$ 3.72). Results indicates that there is a negative correlation between Optimism and suicidal ideation $(\mathrm{M}=33.8$; $\mathrm{SD}=4.94) \mathrm{p}<05^{*}$.

\section{DISCUSSION:}

The present study "Hope Optimism and Suicidal Ideation" was aimed to asses relationship Optimism, Hope and Suicidal Ideation among university students. The findings from the study show that there is no correlation between suicidal ideation and hope. This in consonant with Review that's hope is a potential predictor of low level suicidal ideation in clinical sample (Davidson \& Wingate, 2013). There is inverse correlation between suicidal ideation and optimism which says that higher the optimism scores among the students lower the suicidal ideation, this inference is supported with study done by Duberstein (2007) found that optimism 
negatively predicted suicidal ideation in a college student sample, after accounting for the effects of both depression and hopelessness.

\section{CONCLUSION:}

There is no significant correlation between suicidal ideation and hope among university students. There is a negative correlation between suicidal ideation and optimism which says that higher the optimism scores the lesser the suicidal ideation.

\section{REFERENCES:}

Beck, A. T., Kovacs, M., \& Weissman, A. (1979). Assessment of suicide intention: The Scale for Suicide Ideation. Journal of Consulting and Clinical Psychology, 47, 343-352.

Hirsch, J. K., Conner, K. R., \& Duberstein, P. R. (2007) Optimism and suicide ideation among young adult college students. Archives of Suicide Research, 11, 177-185. doi:10.1080/13811110701249988.

Hirsch, J. K., Wolford, K., LaLonde, S. M., Brunk, L. \& Morris, A. P. (2007). Dispositional optimism as a moderator of the relationship between negative life events and suicide ideation and attempts. Cognitive Therapy and Research, 31, 533-546. doi:10.1007/s10608-007-9151-0

Hirsch, J. K., \& Conner, K. R. (2006). Dispositional and explanatory style optimism as potential moderators of the relationship between hopelessness and suicidal ideation. Suicide and Life-Threatening Behaviour, 36(6), 661-669.

Kumar, S., \& Singh, A. P. (2006). Stress state and its relationship with academic performance among students in A. Husain \& M. I. Khan (Eds.). Recent trends in human stress management (pp. 55-66). New Delhi: Global Vision Publishing House.

Polgreen, Lydia (2010). Suicides, Some for Separatist Cause, Jolt India". The New York Times.

Scheier, M. F. \& Carver, C. S. (1985). Optimism, coping, and health: Assessment and implications of generalized outcome expectancies. Health Psychology, 4, 219-247.

Seligman, M. E. P., \& Csikszentmihalyi, M. (2000). Positive psychology: An introduction. American Psychologist, 55(1), 5-14.

Snyder, C. R. (2002). Hope theory: Rainbows in the mind. Psychological Inquiry, 13(4), 249-275.

The Registrar General of India, Government of India (2012)

Vijaykumar L. (2007), Suicide and its prevention: The urgent need in India, Indian Journal of Psychiatry. 49:81-84,

Wingate, L. R., Burns, A. B., Gordon, K. H., Perez, M., Walker, R. L., Williams, F. M., \& Joiner T. E. (2006). Suicide and positive cognitions: Positive psychology applied to the understanding and treatment on suicidal behavior. In T. E. Ellis, T. E. Ellis (Eds.), Cognition and suicide: Theory, research, and herapy (pp. 261-283). Washington, DC US: American Psychological Association. doi:10.1037/11377-012.

World Health Organization. (2012). Suicide prevention. 